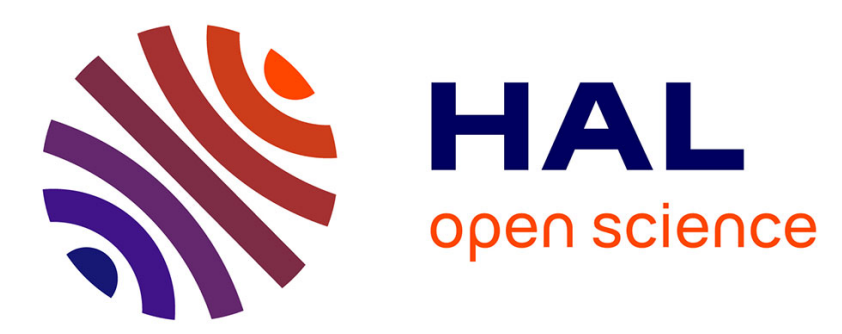

\title{
The basal plane oxygen dependence of YBa2Cu3O7-x anelastic relaxation and orthorhombic to tetragonal phase transition
}

\author{
E. Bonetti, G. Cammarota, E. Campari, A. Casagrande
}

\section{To cite this version:}

E. Bonetti, G. Cammarota, E. Campari, A. Casagrande. The basal plane oxygen dependence of YBa2Cu3O7-x anelastic relaxation and orthorhombic to tetragonal phase transition. Journal de Physique IV Proceedings, 1993, 03 (C7), pp.C7-2033-C7-2038. 10.1051/jp4:19937324 jpa-00251970

HAL Id: jpa-00251970

https://hal.science/jpa-00251970

Submitted on 1 Jan 1993

HAL is a multi-disciplinary open access archive for the deposit and dissemination of scientific research documents, whether they are published or not. The documents may come from teaching and research institutions in France or abroad, or from public or private research centers.
L'archive ouverte pluridisciplinaire HAL, est destinée au dépôt et à la diffusion de documents scientifiques de niveau recherche, publiés ou non, émanant des établissements d'enseignement et de recherche français ou étrangers, des laboratoires publics ou privés. 


\title{
The basal plane oxygen dependence of $\mathrm{YBa}_{2} \mathrm{Cu}_{3} \mathrm{O}_{7-x}$ anelastic relaxation and orthorhombic to tetragonal phase transition
}

\author{
E. BONETTI, G.P. CAMMAROTA*, E.G. CAMPARI and A. CASAGRANDE* \\ Dipartimento di Fisica, Università di Bologna, Consorzio INFM and GNSM-CNR, via Imerio 46, \\ 40126 Bologna, Italy \\ * Istituto di Metallurgia, Università di Bologna, CIRM, v. le Risorgimento 4, 40122 Bologna, Italy
}

\begin{abstract}
Astract- Measurements of the elastic energy dissipation coefficient and of the dynamic Young's modulus were performed on polycrystalline $\mathrm{YBa}_{2} \mathrm{Cu}_{3} \mathrm{O}_{7-\mathrm{x}}$ by the vibrating reed technique and torsional pendulum, in the frequency range $1-5000 \mathrm{~Hz}$. The stoichiometry of the samples was varied in the range $x$ $=0.02-0.7$. From the isothermal modulus measurements a strong softening stage characterizing the onset of the ortho-tetragonal transformation was observed. The long time isotherms give an activation energy of $0.9=0.1 \mathrm{eV}$. Two relaxational damping peaks have been observed and attributed to the short range oxygen dynamics in the well oxygenated and oxygen deficient superconducting phases. On the basis of these results models for the structural transformation linked to oxygen out diffusion and for the oxygen dynamics in different stoichiometric conditions are discussed.
\end{abstract}

\section{I - INTRODUCTION}

It is now well established that oxygen plays a key role in high $\mathrm{T}_{\mathrm{C}}$ superconductivity. The structural stability of the well oxygenated superconducting phase in $\mathrm{YBa}_{2} \mathrm{Cu}_{3} \mathrm{O}_{7-\mathrm{x}}(\mathrm{YBCO})$, is limited by the oxygen out diffusion that occurs predominantly in the $\mathrm{CuO}$ basal plane along the $O(4)$ chain sites $/ 1,2 \%$. Different substoichiometric superconducting phases can be achieved, characterized by different degree of order of the oxygen sublattice sites $/ 3 /$. The orthorhombic to tetragonal transformation occurs finally in dependence of temperature and oxygen partial pressure /4/. The stoichiometry strongly affects the transport properties, the oxygen atoms ordering in the $\mathrm{Cu}-\mathrm{O}$ chains control the hole density in the $\mathrm{Cu}$ basal plane and so affects e.g. the resistivity $/ 5-7 /$. Observations by electron microscopy techniques have indicated that the so called Ortho-II phase is the most stable among the different substoichiometric ones $/ 8 /$. In correspondence to different degree of oxygen vacancy ordering, differences have been observed in the lattice parameter of the elementary cell in the copper basal plane, indicating a directional strain associated to the fraction of occupancy of oxygen in either the $(1 / 200)$ or $(01 / 20)$ sites. In these conditions anelastic relaxation effects can be observed. Experimental results obtained by different research groups have indeed confirmed that in the temperature range where appreciable oxygen mobility is expected, strong relaxational damping maxima can be detected 19-13/. The results obtained by the different authors apart for some similar features show contradictory aspects, for example as regard the number of anelastic relaxation peaks observed. In this research we have extended our previous measurements to get further insight into the microstructural features of the orthorhombic to tetragonal transformation. Moreover the mesurements of the elastic energy dissipation coefficient have been performed in strictly controlled stoichiometric conditions in order to control how the oxygen deficiency affect the damping spectra. Finally we will try to critically compare the results with those obtained by 
other reasearch groups.

\section{II - EXPERIMENTAL}

The measurements were made on polycrystalline YBCO specimens prepared by a solid state reaction procedure /14/. Thin bar shaped specimens obtained by successive consolidation and sintering stages were employed for the anelasticity measurements. A vibrating reed technique with electrostatic drive and frequency modulation detection of flexural vibrations $\left(102-10^{4} \mathrm{~Hz}\right)$, and a torsional pendulum $(1-10 \mathrm{~Hz})$ were employed. All measurements were performed in oxygen partial pressure of $0.5 \mathrm{~Pa}$ and at a maximum strain amplitude below $10^{-5}$. The oxygen content after specimens preparation and following selected thermal treatments, was determined by iodometric titration. Moreover some mesurements were performed also by thermogravimetric (TG) and derivative thermogravimetric (DTG) techniques, at the same heating rates as used in the anelasticity measurements.

\section{III - STRUCTURAL STABILITY AND TRANSFORMATIONS}

Typical Young's modulus vs. time trend, together with a DTG spectrum obtained from a piece of the same original sample are shown in fig.1. A well defined softening stage corresponding to a maximum in the oxygen desorption rate can be observed at $x=0.45-0.5$. According to the Landau theory for a second order phase transition: the behaviour of the modulus as a function of temperature should obey the following relation $/ 15 /$ :

$$
\left.M=M_{0}\left[1+C /\left(T-T_{C}\right)\right]-1 \quad C=\text { cost. } 1\right)
$$

This trend was frequently observed as for example in ferroelastic type transitions /16/. With reference to the phase diagram shown in fig. 2 it is clear that, in isothermal conditions, a progressive oxygen deficiency increase, at around $600 \mathrm{~K}$, lead to a transition from the orthorhombic to the tetragonal phase. The $x$ value determined from the softening stages in our isotherms roughly corresponds to the line that makes a separation between the different phases, thus confirming that the phonon mode softening is indeed linked to the structural transformation. Strictly speaking the observed isothermal modulus behaviour does not show a symmetrical cusp behaviour. The predicted trend can be recovered if one takes into account that oxygen outflow kinetics as can be seen from thermogravimetric measurements are approximately exponentials. In our experimental conditions, at constant temperature and pressure, $x$ is therefore the variable parameter, so with a simple scale change by plotting the normalized dynamic modulus as a function of log-time a cusp type behaviour can be recovered, fig.3.

The long time modulus behaviour,fig.4 can be described as previously reported $/ 17 /$, by the following expression:

$$
\left(\mathrm{M}-\mathrm{M}_{\mathrm{O}}\right) /\left(\mathrm{M}-\mathrm{M}_{\mathrm{O}}\right)=[1-\exp (-\mathrm{t} / \tau)]
$$

with $\tau=\tau_{0} \exp (\mathrm{H} / \mathrm{kT})$, from 2$)$ a value $H=(0.9+0.1) \mathrm{eV}$ was derived $/ 17 /$.

The overall behaviour displayed by the isothermal and isochronal modulus trends, figs.1,4, reflect a structural transformation triggered by the oxygen depletion (out diffusion), during which different processes occur. Phonon mode softening is generally speaking a natural precursor of structural transformations. The strong softening observed at our partial oxygen pressure for temperatures in excess of $600 \mathrm{~K}$ in nearly stoichiometric samples, reflect an elastic instability that underwent at oxygen deficiency $x=0.4-0.5$. As indicated by TEM observations a prominent structural feature characterizing the fully oxygenated stoichiometric 123 YBCO phase are the twins $/ 4,18 /$. They form on (110) planes as a result of the mismatch between the lattice parameters and originate from the arrangement of oxygen vacant sites in the $\mathrm{Cu}-\mathrm{O}$ planes. This arrangement is a way to minimize the strain energy in the 123 phase when the 


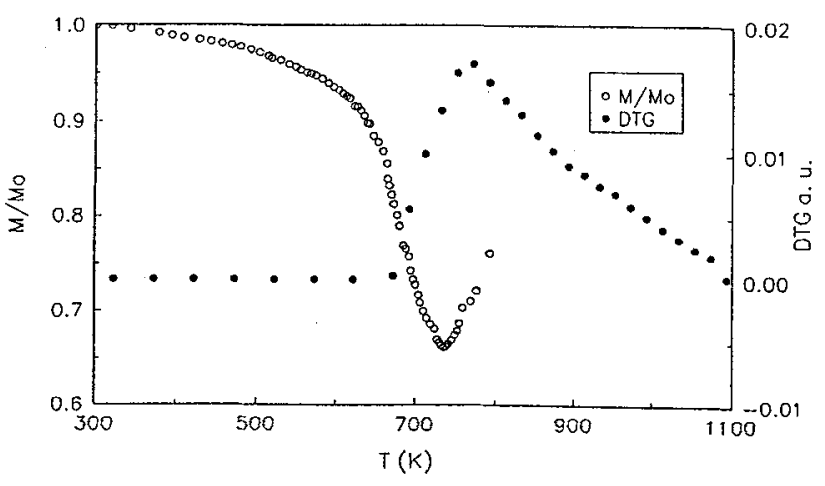

Fig.1 Dynamic Young's modulus and DTG trends vs. temperature for a specimen just as prepared $(x=0,07)$. Heating rate $2 \mathrm{~K} / \mathrm{min}$. Mo: modulus at room temperature.

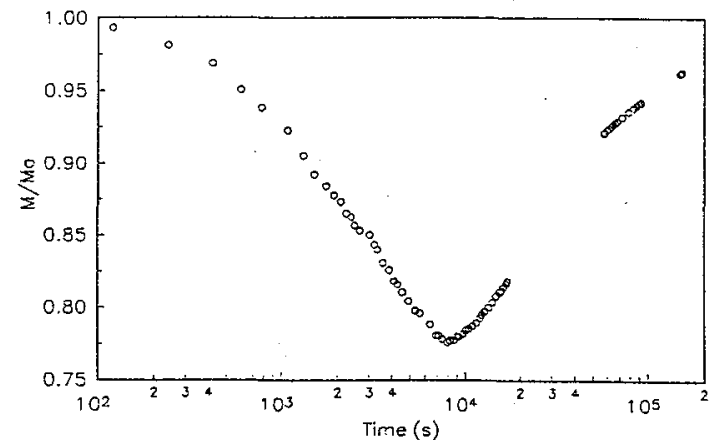

Fig.3 Softening of the dynamic Young's modulus during an isothermal measurement at $705 \mathrm{~K}$. Note the inverse cusp behaviour as predicted by eq.1. In this case $x$ (i.e $t$ ) is the variable parameter.
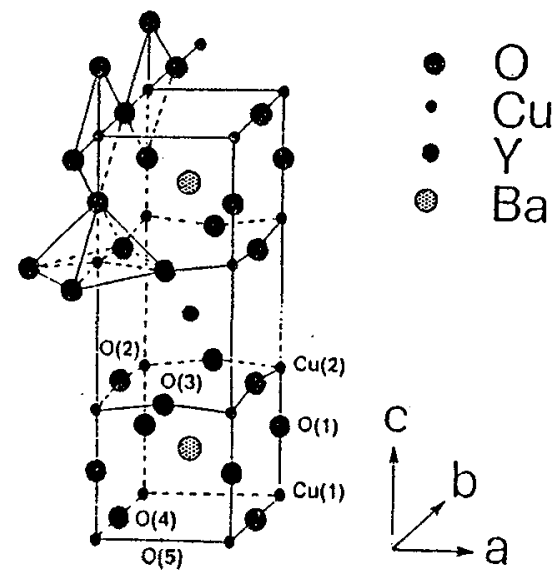

a)

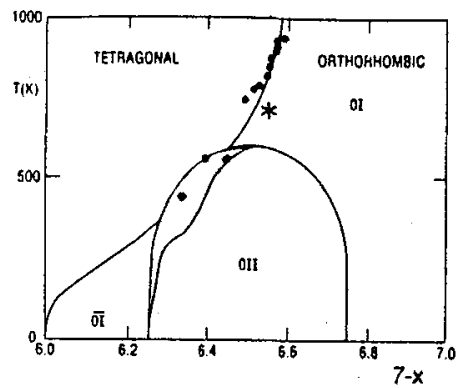

b)

Fig.2 a) YBCO structure. Oxygen and Copper atoms in the chains are labelled $\mathrm{O}(4), \mathrm{Cu}(1)$. Those of superconducting planes by $\mathrm{O}(2), \mathrm{O}(3)$ and $\mathrm{Cu}(2)$. b) $\mathrm{YBCO}$ phase diagram. From refs. 23,25.

Fig.4 Isothermal trends of the dynamic Young's modulus and the elastic energy dissipation coefficient. Temperature $680 \mathrm{~K}$. Frequency $1 \mathrm{KHz}$.

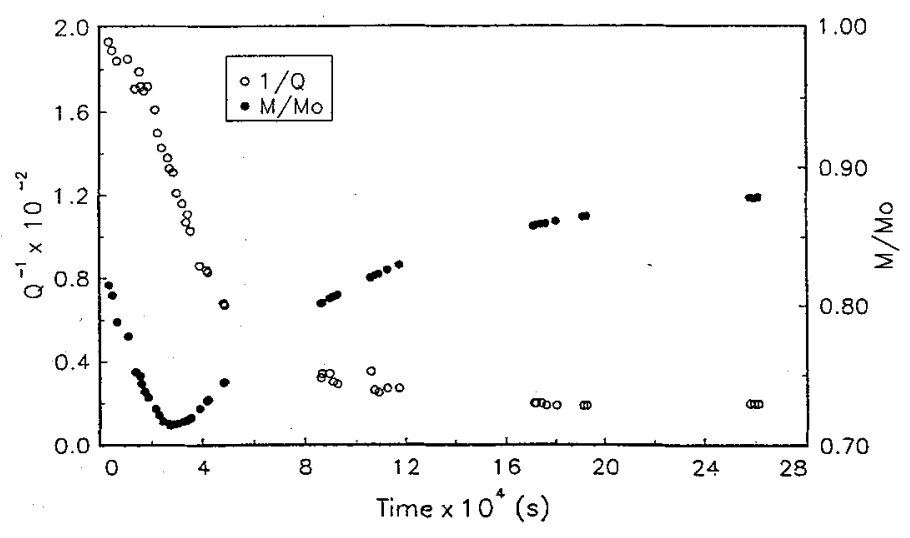


tetragonal to orthorhombic transformation occurs. The experimental observations indicate a reduction in the twins number and area following oxygen out diffusion at oxygen deficiency $\mathrm{x}=0.3-0.5$.

So the strong softening stage, corresponds to an enhanced oxygen desorption rate in the orthorhombic well oxygenated phase $(x=0.02-0.08)$ probably achieved through preferential channels such as the twinned interfaces, whose resultant high mobility give rise to strong anelastic effects, followed in the temperature scale by the twins area e/o number reduction, like in ferroelastic type transitions $/ 19 /$.

The long annealing type behaviour leading to a modulus increase, with a further very low reduction in oxygen content should reflect an order -disorder transition in the $\mathrm{Cu}(1)$-O(4) chains of the remaining orthorhombic microdomains embedded in a tetragonal habit $/ 17 /$.

\section{IV - ANELASTIC RELAXATION AND OXYGEN SHORT RANGE DYNAMICS}

Measurements of the elastic energy dissipation have been extensively applied recently to study oxygen mobility and diffusion in $\mathrm{YBCO} / 10-13,20 /$. One of the main point to be adressed, and up to now not completely clarified, regards the number and the nature of thermally activated relaxation processes, observed in the temperature range where appreciable oxygen mobility sets up . The first measurements performed by Berry $/ 12 /$, by the vibrating reed technique showed a well defined anelastic relaxation peak $(600 \mathrm{~K}, 350 \mathrm{~Hz})$, attributed to orientational ordering of oxygen atoms. Measurements performed subsequently by different experimental groups, have confirmed that strong anelastic relaxation effects depending on oxygen may be experienced above room temperature at acoustic frequencies. These measurements have moreover indicated that there is a measurable stoichiometry effect upon the relaxation time $\tau$ characterizing the relaxation processes, i.e a peak temperature shift by varying the oxygen content in the superconducting orthorhombic phase. This is an unwanted circumstance if one desire to accurately determine the anelastic relaxation parameters because measurements in strictly controlled stoichiometric conditions are needed. Another problem at present unresolved regards the number of relaxation peaks: some authors report only one peak attributed to oxygen relaxation $/ 10,12,13,21 /$ other authors report the simultaneous presence in the spectrum of two internal friction peaks $/ 9,11,22 /$.

In our measurements, in nearly stoichiometric conditions $(x=0.02-0.07)$ an anelastic relaxation peak, previously labelled $\mathrm{P}_{\mathrm{O}} 1$ is observed, Fig.5/11,22/. At $\mathrm{KHz}$ frequencies the peak temperature just preceeds the modulus instability regime above discussed (section III), only with measurements performed at lower frequencies, to shift the peak at lower temperature, the intrinsic anelastic nature of this peak can be clearly evinced. The measured activation energy is $\mathrm{H}_{\mathrm{O} 1}=(1.5+0.1) \mathrm{eV} / 24 /$. The peak relaxation strenght strongly decreases with increasing the oxygen deficiency strongly suggesting a relaxation mechanism involving oxygen dynamics in the superconducting Ortho-I phase. A second peak, previously labelled $\mathrm{P}_{\mathrm{O} 2}$ was observed at significantly lower temperatures in substoichiometric superconducting samples $(x>0.1-0.2)$ Fig.6, the corresponding activation energy is $\mathrm{H}_{\mathrm{o} 2}=(1.3+0.1) \mathrm{eV}$, this value is very close to the one deduced recently by Woirgard et al. /21/ for a peak observed by isothermal measurements. The maximum in the relaxation strenght for $\mathrm{P}_{\mathrm{O} 2}$ occurs at approximately $\mathrm{x}=0.4-0.5$, in agreement with the peak height increase with $x$ up to $x=0.46$ reported by $X i e / 10 /$. Both peaks can be observed in the internal friction spectrum for intermediate oxygen deficiency values $x=$ 0.1-0.3, Fig.5. The experimental data regarding the relaxation time are reported in Fig.7, together with those obtained by different experimental groups and recently reviewed by Cost $/ 20 /$. The peak temperature shift observed by Berry /12/ of the order of $20 \mathrm{~K}$, by varying the oxygen content with prolonged vacuum annealing, strongly suggest a stoichiometry effect on the relaxation time $\tau$. A similar stoichiometric effet was previously observed, by measurements at lower frequencies by $\mathrm{Xie} / 10$ / who determined a $20 \mathrm{~K}$ peak temperature shift by increasing $x$ from 0.12 to 0.46 . This is reasonably the reason why the plot in Fig.6, show a wide scatter of the experimental data particularly evident for the measurements at temperatures higher than about 
Fig.5 Internal friction vs. temperature for two YBCO specimens with different oxygen contents $(7-x)$ as indicated. Note the $P_{01}$ height decrease and $P_{02}$ appearance at $x=0.1$. Vibration frequency $1.5 \mathrm{KHz}$.
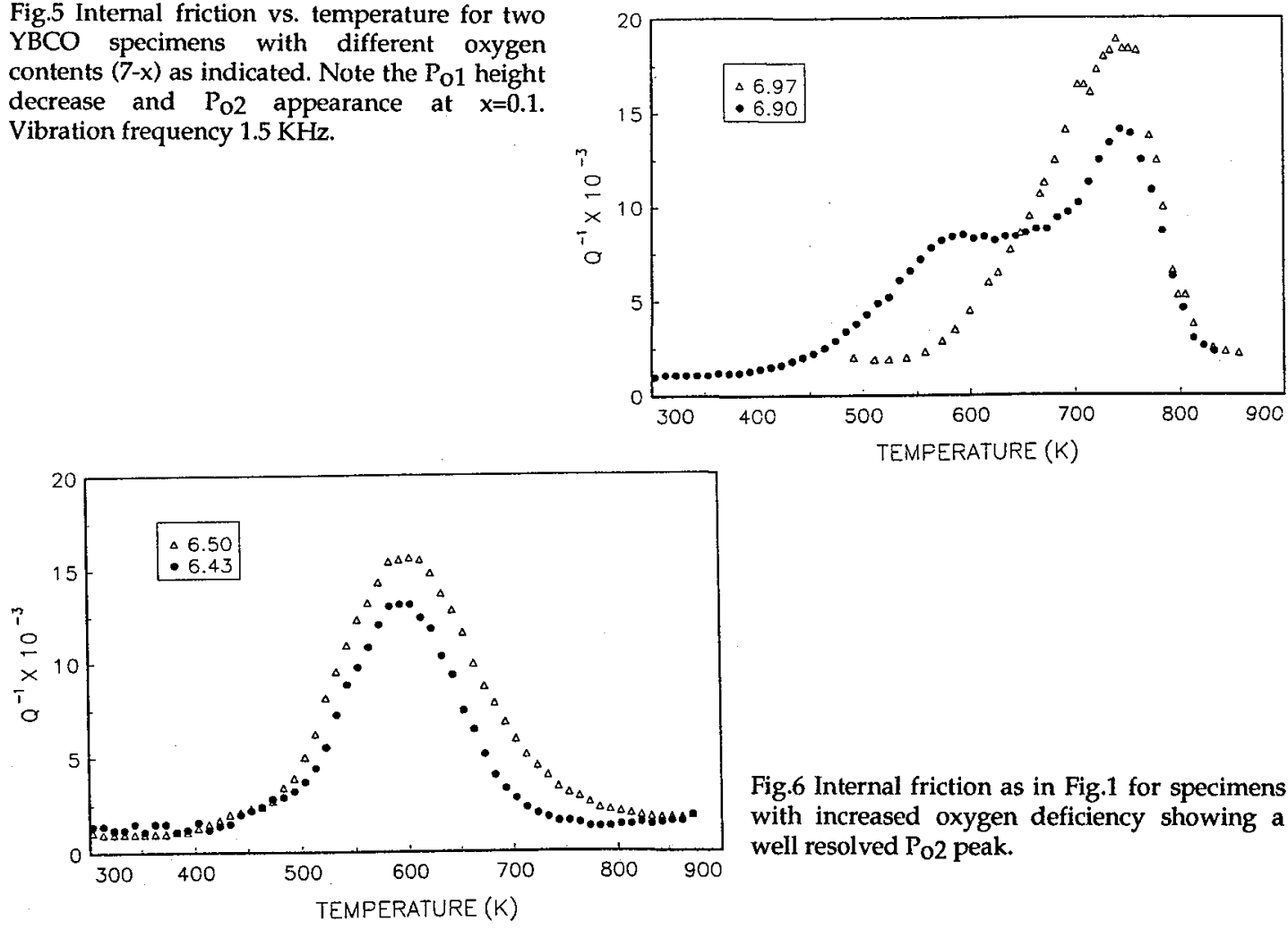

Fig.6 Internal friction as in Fig.1 for specimens with increased oxygen deficiency showing a well resolved $\mathrm{P}_{02}$ peak.

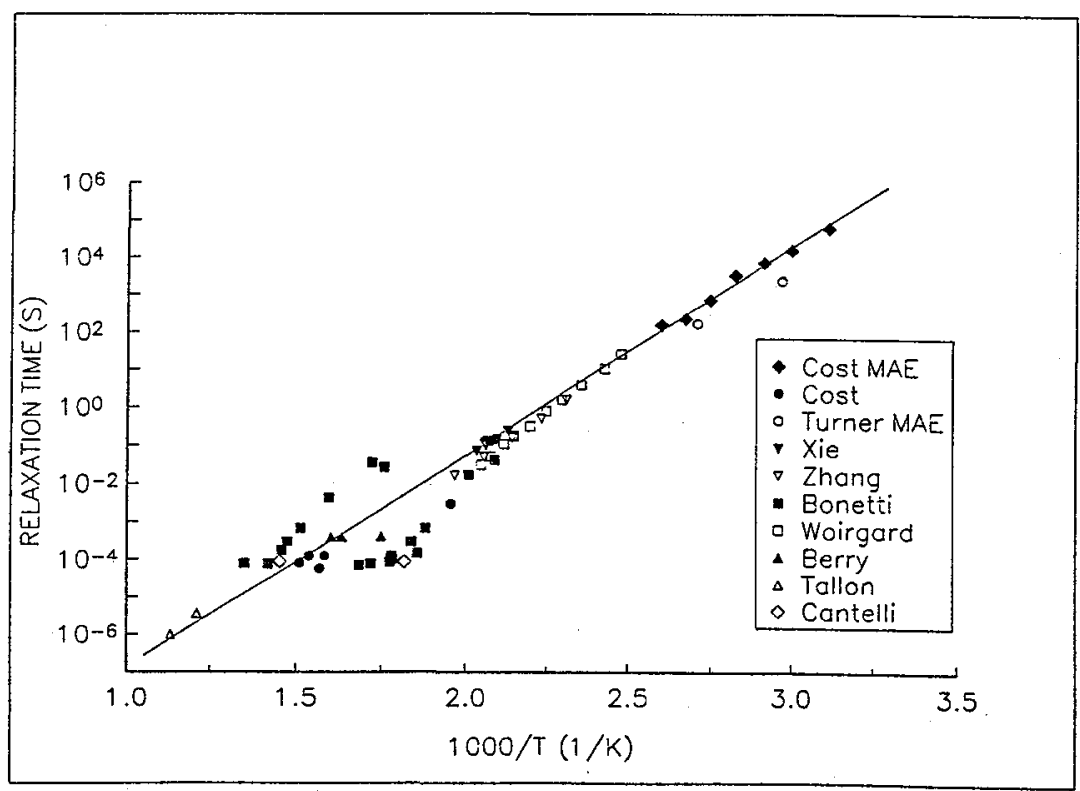

Fig.7 Arrhenius plot of the relaxation time for oxygen relaxation measured by internal friction and in two cases by mechanical after effect (MAE) by different authors. 
$480 \mathrm{~K}$. In fact in the most frequently employed experimental conditions (partial pressure) at these temperatures some oxygen outflow during measurements may occur.

On the other hand, the points referring to our $\mathrm{P}_{\mathrm{O} 1}, \mathrm{P}_{\mathrm{O} 2}$ peaks and to the $\mathrm{P}_{\mathrm{H} 1}, \mathrm{P}_{\mathrm{H} 2}$ peaks from Cannelli et al. $/ 22 /$, belongs apparently to two groups well separated,(more than $100 \mathrm{~K}$ apart at $\mathrm{KHz}$ frequencies). So it seems realistic assume they correspond to distinct relaxation mechanisms involving oxygen in substantially different environmental habits e.g those corresponding to the Ortho-I and Ortho-II phases.

The higher activation energy for peak $\mathrm{P}_{01}$, may result from the higher number of nearest $\mathrm{O}_{4}$ oxygen atoms around the $\mathrm{O}_{5}$ sites in the Ortho-I phase that, on account of the positive interaction energy between $O(4)$ and $O(5) / 25 /$, should lead to a higher barrier for the $O$ jumps between the $(01 / 20)$ and $(1 / 200)$ sites. Up to now the alternative hypothesis already proposed $/ 11 /$, that $P_{01}$ is due to anelastic relaxation process involving the twinned interfaces cannot be excluded.

\section{REFERENCES}

1. J.D.Jorgensen, B.W.Veal, A.P.Paulikas, L.J.Nowicki, G.B.Crabtee, H.Claus, W.K.Kwok, Phys. Rev.B 41 (1990) 1863.

2. W.K.Kwok, G.W.Krabtee, A.Umezawa, B.W.Veal, J.D.Jorgensen, S.K.Malik, L.J.Nowicki, A.P.Paulikas, L.Nunez, Phys. Rev. B, 37 (1988) 106.

3. O.R.Monteiro, J.Evans, S.M.Johnson, J.Appl.Phys., 69 (1991) 2414.

4. J.H.Muller,W.Mertin, and R.Gruehn, Supercond.Sci.Technol., 3 (1990) 273.

5. K.N.Tu, N.C.Yeh, S.I.Park and C.Tsuei, Phys. Rev.B, 39 (1989) 304.

6. K.A.Muller, PhysicaC, 185-189 (1991) 3.

7. S.Lapinskas, A.Rosengreen, E.E.Tornau, Physica C, 199 (1992) 91.

8. G. van Tendeloo, D.Broddin, H.W.Zandbergen, S.Amelinckx, Phisica C, 167 (1990) 627.

9. J.X.Zhang, G.M.Lin, Z.C.Lin, K.F.Liang, P.C.W.Fung, and G.G.Siu, J.Phys. C, Condens. Matter, 1 (1989) 6939.

10. X.M.Xie, T.G.Chen and Z.L.Wu, Phys. Rev.B, 40 (1989) 4549.

11. E.Bonetti, E.G.Campari, G.P.Cammarota, A.Casagrande, and S.Mantovani, J.Less Comm. Metals, 164-5 (1990) 231

12. B.S.Berry, W.C.Pritchet and T.M.Shaw, Defect and Diffusium Forum, 75. (1991) 34.

13. J.R.Cost and J.T.Stanley, J.Mater. Res., 6 (1991) 232.

14. E.Bonetti, E.G.Campari, G.P.Cammarota, A.Casagrande and G.Centi, Proc. II EUROMAT, Cambridge, July 1991.

15. A.S.Nowick and B.S.Berry, Anelastic relaxation in crystalline solids, Academic Press, New York 1972, pp.464-492.

16. G.Erradonea, J.Physique, C6-42 (1981) 710.

17. E.Bonetti, E.G.Campari, T.Manfredini and S.Mantovani, Physica C, 179 (1991) 381.

18. G.Van Tendeloo, S.Amelinkx, J.Less.Comm. Metals, 164-165 (1990) 92.

19. T.Hatanaka and A. Sawada, Jap. J. Appl. Phys., 28 (1989) L-392.

20. J.R.Cost and J.T.Stanley, Mater. Science Forum, 119-121 (1993) 623.

21. J.Woirgard, A.Riviere, P.Gadaud et P.Tal, Europhys. Lett., 17 (1992) 601.

22. G.Cannelli, R.Cantelli, F.Cordero, and F.Trequattrini, Supercond. Sci. Technol., 5 (1992) 247.

23. E.Bonetti, E.G.Campari and S.Mantovani, Physica C, 196 (1992) 7.

24. E.Bonetti, E.G.Campari, V.Luprano, S.Mantovani, S.Casagrande and G.P.Cammarota, Mater. Science Forum, 119-121 (1993) 689.

25. D.de Fontaine, Oxygen Disorder Effects in High- $\mathrm{T}_{\mathrm{C}}$ Superconductors, J.Moran and I.Shuller eds., Plenum, New York 1990. 